\title{
Singlet excited-state absorption of eosin Y
}

\author{
Alfons Penzkofer, Ali Beidoun \\ Naturwissenschaftliche Fakultät II - Physik, Universität Regensburg, W-8400 Regensburg, Germany \\ and \\ Shammai Speiser \\ Department of Chemistry, Technion - Israel Institute of Technology, Technion City, Haifa 32 000, Israel
}

Received 19 August 1992

\begin{abstract}
The absolute $\mathrm{S}_{1}$-state excited-state absorption cross-section spectrum of eosin $\mathrm{Y}$ in methanol has been determined by picosecond second harmonic $\mathrm{Nd}$ : glass laser excitation and time-delayed picosecond light continuum probing (probing wavelength region from 400 to $980 \mathrm{~nm}$ ).
\end{abstract}

\section{Introduction}

The excitation of matter using intense ultrashort light pulses causes a transient change of the optical constants [1-12] (reduction of ground-state absorption, creation of stimulated emission and excited-state absorption, transient refractive index changes, optically induced dichroism and birefringence). The excitation with an intense ultrashort pump pulse and the probing with a time-delayed ultrashort white-light continuum allows a time resolved spectroscopic analysis of transient optical phenomena $[10,12,13]$. In laser materials the excited-state absorption in the pump wavelength region reduces the pumping efficiency, and the excited-state absorption in the stimulated emission wavelength region reduces the laser gain $[14,15]$ and the laser tuning range [16]. If the excited-state absorption cross section is larger than the stimulated emission cross section, then no laser action is possible. In mode-locking dyes the excitedstate absorption reduces the saturable absorber action (bleaching efficiency is reduced) [17]. In wavelength regions where the excited-state absorption is larger than the ground-state absorption, reverse saturable absorption occurs [17-22]. Time-resolved measurements of excited-state absorption may give valuable information on the photochemical dynam- ics of electronically excited matter $[4,5,8]$.

In this paper we determine the absolute singlet-state excited-state absorption cross-section spectrum of the organic dye eosin $Y$ (disodium salt of $2^{\prime}, 4^{\prime}, 5^{\prime}, 7^{\prime}$. tetrabromofluorescein, structural formula is shown in fig. 6 ) in the organic solvent methanol at room temperature. Eosin $\mathrm{Y}$ in various solvents has been applied as laser dye [23]. It is an important stain in histology [24] and was recently used for optical bistability [25] and in all-optical switching schemes [26]. A picosecond pump and probe technique $[1,2,10,12]$ is applied in the experiments using frequency doubled pulses of a mode-locked Nd:glass laser [27] for excitation and a picosecond light continuum [28] following the pump pulse for probing. The excited-state absorption cross-section spectrum in the wavelength region between 400 and $980 \mathrm{~nm}$ is determined. A comparison of the excited-state absorption cross-section spectrum with the stimulated emission cross-section spectrum determines the range of possible laser action, and a comparison of the excited-state absorption cross-section spectrum with the ground-state absorption cross-section spectrum locates the regions of saturable absorber action and reverse saturable absorber action. 


\section{Experimental}

The experimental pump-probe setup is shown in fig. 1. An active (acousto-optic modulator IntraAction model ML-50Q) and passive (saturable absorber is Kodak dye No. 9860 in 1,2-dichlorethane) mode-locked Nd:phosphate glass laser (Schott laser glass type LG760) generates a train of picosecond light pulses at a wavelength of $1054 \mathrm{~nm}$. A single pulse is selected from the pulse train by an electro-optic shutter [29] and increased in energy in a $\mathrm{Nd}$ : phosphate glass amplifier. Single pulse energies up to about $5 \mathrm{~mJ}$ are generated. The laser may be operated from single-shot up to $0.05 \mathrm{~Hz}$ repetition rate. The second harmonic light is generated in a CDA crystal [30] (cesium dihydrogen arsenate Cs$\mathrm{H}_{2} \mathrm{AsO}_{4}$, length $1 \mathrm{~cm}$, noncritical $90^{\circ}$ phase-matching at $34^{\circ} \mathrm{C}$ ). Second harmonic energy conversion efficiencies of 30 to $40 \%$ are obtained. The second harmonic pulse duration is $\Delta t_{\mathrm{L}} \approx 5 \mathrm{ps}$.

Behind the CDA crystal the fundamental laser pulse and the second harmonic pulse are separated by a harmonic beam splitter. The fundamental laser pulse is filtered, passed through an optical delay line, and focused to a heavy water cell where the picosecond light continuum is generated [28]. Behind the heavy water cell the fundamental laser light is filtered off (filter F3) and the white light continuum is imaged to the sample cell $\mathrm{S}$. Part of the light continuum is directed to the spectrometer SPI where the input spectrum is recorded (diode array system DA). The transmitted light continuum behind the eosin $Y$ sample is directed to the spectrometer SP2 where the output spectrum is recorded (vidicon system VI). The input and output spectra are digitized and transferred to a computer for data analysis.

The second harmonic light which passes the harmonic beam splitter serves as an excitation pulse of the eosin $Y$ sample. The pump pulse transmission through the sample is determined with the photodetectors PD1 and PD2. The half-wave plate WP is aligned to adjust the polarization of pump pulse to the magic angle $\left(\theta=54.7^{\circ}\right)$ relative to the polarization of the linearly polarized probe pulse continuum. The magic angle alignment avoids complications of anisotropic probe pulse absorption caused by anisotropic molecular orientation due to electric dipole interaction $[1,31]$. The optical delay line DL in the probe pulse branch is adjusted to an optical delay of $t_{\mathrm{d}} \approx 30 \mathrm{ps}$ of the probe pulse behind the pump pulse. This delay time is short compared to the fluorescence lifetime, and intersystem crossing to a triplet state is negligible for this short time delay [32]. The probe continuum transmission is determined only by the $S_{1}$ -

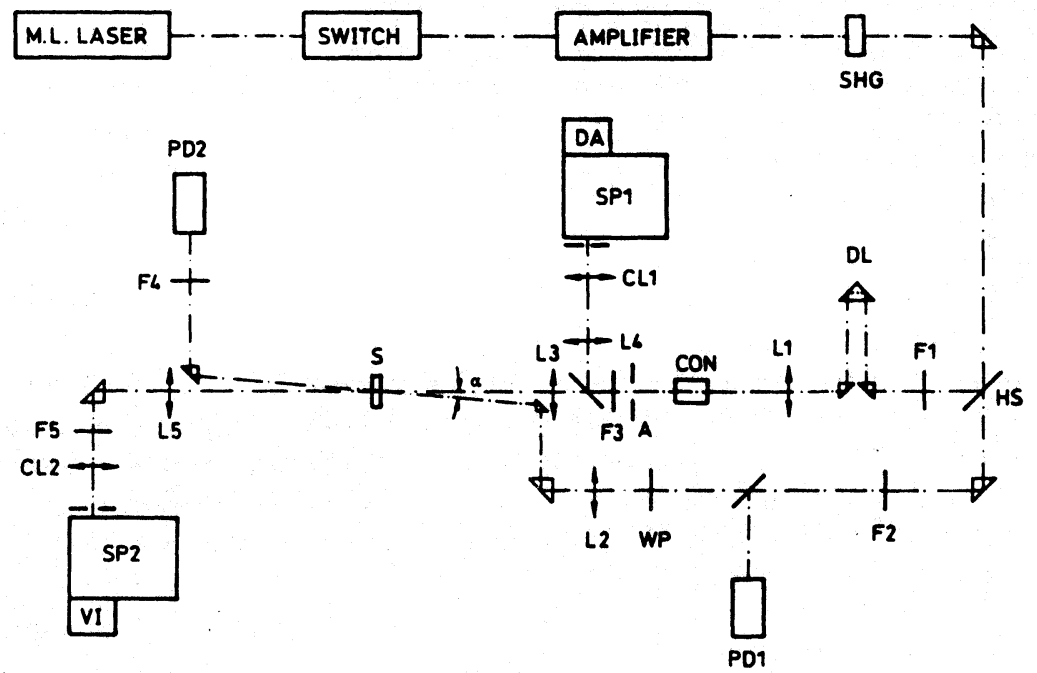

Fig. 1. Experimental setup. SHG, CDA crystal for second harmonic generation. HS, harmonic beam splitter. F1-F5, filters. L1-L5, spherical lenses. CL1, CL2, cylindrical lenses. DL, optical delay line. CON, heavy water cell for picosecond light continuum generation. S, dye sample. PD1, PD2, photodetectors. A. aperture. WP, half-wave plate for magic angle adjustment. Angle between pump and probe beam, $\alpha \approx 3^{\circ}$. SP1, SP2, $25 \mathrm{~cm}$ grating spectrometers with 150 lines $/ \mathrm{mm}$ gratings. DA, diode array system. VI, vidicon system. 
state population (excited-state absorption and stimulated emission) and the residual $S_{0}$-ground state population (ground-state absorption). The $S_{1}$-state population is calculated from the pump pulse transmission (see below).
The dye eosin $Y$ in methanol is investigated at room temperature. Eosin $Y$ was purchased from Heraeus and was purified by recrystallization six times from ethanol.

\section{Theory}

The energy level system of the dye together with the pump pulse and probe pulse transitions and the level depopulations are shown in fig. 2 . The pump pulse absorption dynamics determines the $S_{1}$-state level population as a function of time, and the probe pulse transmission allows the determination of excited-state absorption as a function of wavelength. In a previous paper on intersystem crossing [32] a differential equation system for the level populations and the light pulse propagations was given. Here, in the following equation system triplet level population is neglected (delay time of probe pulse is short compared to inverse intersystem crossing rate) and amplified spontaneous emission is not included since it is negligible for our experimental conditions (see below).

The equation system for the pump pulse passage through the sample reads $[32,33]$ :

$$
\frac{\delta N_{1}(\theta)}{\partial t^{\prime}}=-3 \sigma_{\mathrm{L}} \cos ^{2} \theta\left[N_{1}(\theta)-N_{2}(\theta)\right] \frac{I_{\mathrm{L}}}{h \nu_{\mathrm{L}}}+\frac{N_{2}(\theta)+N_{2^{*}}(\theta)}{\tau_{\mathrm{F}}}-\frac{N_{1}(\theta)-\bar{N}_{1}}{\tau_{\text {or }}},
$$

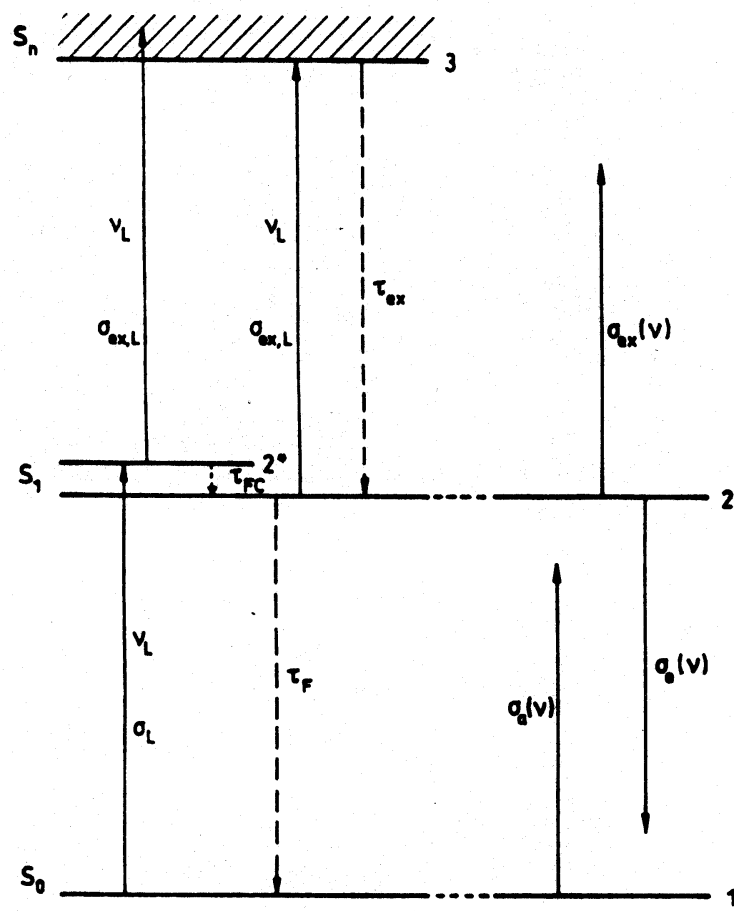

$|a|$
Fig. 2. Energy level diagram of eosin Y. (a) Transitions involved in $\mathrm{S}_{1}$-state level population by pump pulse. (b) Absorption and emission processes involved in time-delayed probe continuum passage. 


$$
\begin{aligned}
& \frac{\partial N_{2^{*}}(\theta)}{\partial t^{\prime}}=\left[3 \sigma_{\mathrm{L}} \cos ^{2} \theta\left[N_{\mathrm{L}}(\theta)-N_{2^{*}}(\theta)\right]-\sigma_{\mathrm{ex}, \mathrm{L}}\left(N_{2^{*}}(\theta)-\frac{N_{2^{*}}(\theta)}{N_{2}(\theta)+N_{2^{*}}(\theta)} N_{3}(\theta)\right)\right] \frac{I_{\mathrm{L}}}{h \nu_{\mathrm{L}}} \\
& \quad-\frac{N_{2^{*}}(\theta)-N_{2^{*}, \mathrm{th}}(\theta)}{\tau_{\mathrm{FC}}}-\frac{N_{2^{*}}(\theta)}{\tau_{\mathrm{F}}}-\frac{N_{2^{*}}(\theta)-\bar{N}_{2^{*}}}{\tau_{\mathrm{or}}}, \\
& \frac{\partial N_{2}(\theta)}{\partial t^{\prime}}=\frac{N_{2^{*}}(\theta)-N_{2^{*}, \mathrm{~h}}(\theta)}{\tau_{\mathrm{FC}}}-\sigma_{\mathrm{ex}, \mathrm{L}}\left(N_{2}(\theta)-\frac{N_{2}(\theta)}{N_{2}(\theta)+N_{2^{*}}(\theta)} N_{3}(\theta)\right) \frac{I_{\mathrm{L}}}{h \nu_{\mathrm{L}}} \\
& \quad+\frac{N_{3}(\theta)}{\tau_{\mathrm{ex}}}-\frac{N_{2}(\theta)}{\tau_{\mathrm{F}}}-\frac{N_{2}(\theta)-\bar{N}_{2}}{\tau_{\mathrm{or}}}, \\
& \frac{\partial N_{3}(\theta)}{\partial t^{\prime}}=\sigma_{\mathrm{ex}, \mathrm{L}}\left[N_{2^{*}}(\theta)+N_{2}(\theta)-N_{3}(\theta)\right] \frac{I_{\mathrm{L}}}{h \nu_{\mathrm{L}}}-\frac{N_{3}(\theta)}{\tau_{\mathrm{ex}}}-\frac{N_{3}(\theta)-N_{3}}{\tau_{\mathrm{or}}}, \\
& \frac{\partial I_{\mathrm{L}}}{\partial z^{\prime}}=-3 \sigma_{\mathrm{L}} I_{\mathrm{L}} \int_{0}^{\pi / 2}\left[N_{1}(\theta)-N_{2^{*}}(\theta)\right] \cos ^{2} \theta \sin \theta \mathrm{d} \theta-\sigma_{\mathrm{ex}, \mathrm{L}} I_{\mathrm{L}}\left[N_{2^{*}}(\theta)+N_{2}(\theta)\right], \\
& \vec{N}_{i}=\int_{0}^{\pi / 2} N_{i}(\theta) \sin \theta \mathrm{d} \theta, \quad i=1,2,2^{*}, 3, \\
& N_{2^{*}, \mathrm{th}}(\theta)=N_{2}(\theta) \exp \left[-\frac{h \Delta \nu_{2,2^{*}}}{k_{\mathrm{B}} \theta}\right] .
\end{aligned}
$$

The transformations $t^{\prime}=t-c_{0} z / n$ and $z^{\prime}=z$ are used, where $t$ is the time, $z$ is the distance along the propagation direction, $c_{0}$ is the light velocity in vacuum, and $n$ is the refractive index. The absorption anisotropy of the electric dipole interaction is considered by the angle dependent ground-state absorption cross section $\sigma_{\mathrm{L}}(\theta)=3 \sigma_{\mathrm{L}} \cos ^{2} \theta$ [31] where $\theta$ is the angle between the transition dipole moment of the molecules and the direction of the electric field of the linearly polarized excitation light. The weak $S_{1}$-excited-state absorption of the pump laser light is approximated by an isotropic absorption, i.e. $\sigma_{\mathrm{ex}, \mathrm{L}}(\theta)=\sigma_{\mathrm{ex}, \mathrm{L}}$ is used in the equations (for a discussion see ref. [32]). The generated angularly anisotropic level populations $N_{i}(\theta)$ relax to isotropic distributions $\bar{N}_{i}$ with the molecular reorientation time $\tau_{\text {or }}$. The depopulation of the $S_{1}$ state $\left(2\right.$ and $\left.2^{*}\right)$ is characterized by the fluorescence lifetime $\tau_{\mathrm{F}}$. The excited Franck-Condon level $2^{*}$ relaxes to the temporal thermal equilibrium state 2 in the $S_{1}$ band with the Franck-Condon relaxation time constant $\tau_{\mathrm{FC}}$. The thermal population $N_{2^{*}, \text { th }}$ of the level $2^{*}$ within the $\mathrm{S}_{1}$ band is taken into account by eq. (7) where $h$ is the Planck constant, $\Delta \nu_{2,2^{*}}$ is the frequency difference between the levels $2^{*}$ and $2, k_{\mathrm{B}}$ is the Boltzmann constant and $\theta$ is the temperature. Molecules are excited from the $S_{1}$ state to a higher lying singlet state (level 3 ) by excited-state absorption (absorption cross section $\left.\sigma_{\mathrm{ex}, \mathrm{L}}\right)$, and they relax back to the $S_{1}$ state with the time constant $\tau_{\mathrm{ex}}$.

The initial conditions of the level populations are $N_{1}\left(\theta, t^{\prime}=-\infty, z^{\prime}, r\right)=N_{0}, N_{2}\left(\theta, t^{\prime}=-\infty, z^{\prime}, r\right)=N_{2^{\bullet}}(\theta$, $\left.t^{\prime}=-\infty, z^{\prime}, r\right)=N_{3}\left(\theta, t^{\prime}=-\infty, z^{\prime}, r\right)=0 . N_{0}$ is the total number density of dye molecules. $r$ is the radial coordinate. The input pump laser intensity is

$I_{\mathrm{L}}\left(t^{\prime}, z=0, r\right)=I_{0 \mathrm{~L}} \exp \left[-\left(\frac{t^{\prime}}{t_{\mathrm{L}}}\right)^{2}-\left(\frac{r}{r_{\mathrm{L}}}\right)^{2}\right]$,

where $I_{0 L}$ is the input peak intensity, $t_{L}=2^{-1}(\ln 2)^{-1 / 2} \Delta t_{L}$ is half the $1 / e$ temporal pulse width $\left(\Delta t_{L}\right.$ is the fwhm pulse duration ), and $r_{\mathrm{L}}$ is the $1 / \mathrm{e}$ beam radius.

The energy transmission $T_{\mathrm{E}, \mathrm{L}}$ of the pump pulse is

$$
T_{\mathrm{E}, \mathrm{L}}=\frac{\int_{0}^{\infty} r \int_{-\infty}^{\infty} I_{\mathrm{L}}\left(t^{\prime}, l, r\right) \mathrm{d} t^{\prime} \mathrm{d} r}{\int_{0}^{\infty} r \int_{-\infty}^{\infty} I_{\mathrm{L}}\left(t^{\prime}, 0, r\right) \mathrm{d} t^{\prime} \mathrm{d} r}
$$


where $l$ is the sample length. The length averaged and orientation averaged level populations at the time position $t_{\mathrm{d}}$ of probe pulse passage are

$\bar{N}_{i, l}\left(t_{\mathrm{d}}, r\right)=\frac{1}{l} \int_{0}^{l} \bar{N}_{i}\left(t_{\mathrm{d}}, z^{\prime}, r\right) \mathrm{d} z^{\prime} . \quad i=1,2,2^{*}, 3$.

For $t_{\mathrm{d}} \gg \tau_{\mathrm{ex}}$ only the levels 1,2 and $2^{*}$ possess remarkable populations.

The solution of the eqs. (1)-(10) provides the energy transmission $T_{\mathrm{E}, \mathrm{L}}$ and the length and orientation averaged level populations.

The length and orientation averaged $\mathrm{S}_{1}$-state level population $N_{\mathrm{S} 1, l}\left(r, t_{\mathrm{d}}\right)=\bar{N}_{2, l}\left(r, t_{\mathrm{d}}\right)+\bar{N}_{2^{*}, l}\left(r, t_{\mathrm{d}}\right)$ determines the magic-angle probe pulse transmission through the sample at time $t_{\mathrm{d}}$. The initial intensity distribution of the probe pulse spectrum is

$I_{\mathrm{P}}\left(\nu, r, t^{\prime}, z^{\prime}=0\right)=I_{\mathrm{P}, 0}(\nu) \exp \left(-\frac{r^{2}}{r_{\mathrm{P}}^{2}}-\frac{\left(t^{\prime}-t_{\mathrm{d}}\right)^{2}}{t_{\mathrm{P}}^{2}}\right)$,

where $r_{\mathrm{p}}$ is the $1 / \mathrm{e}$ probe beam radius, and $t_{\mathrm{p}}$ is half the $1 / \mathrm{e}$ probe pulse duration. The probe pulse energy is kept small so that population changes caused by the probe pulse are negligible. The angle between the polarization direction of the probe pulse continuum and the polarization direction of the pump pulse is adjusted to the magic angle in order to avoid anisotropy effects [31]. Under these conditions the probe pulse propagation through the sample is given by

$$
\begin{aligned}
& \frac{\partial I_{\mathrm{P}}\left(\nu, r, t^{\prime}\right)}{\partial z^{\prime}}=\left\{-\sigma_{\mathrm{a}}(\nu) \bar{N}_{1, l}\left(r, t_{\mathrm{d}}\right)+\left[\sigma_{\mathrm{e}}(\nu)-\sigma_{\mathrm{ex}}(\nu)\right] \bar{N}_{\mathrm{S} 1, l}\left(r, t_{\mathrm{d}}\right)\right\} I_{\mathrm{P}}\left(\nu, r, t^{\prime}\right) \\
& \quad=\left\{-\sigma_{\mathrm{a}}(\nu) N_{0}+\left[\sigma_{\mathrm{a}}(\nu)+\sigma_{\mathrm{e}}(\nu)-\sigma_{\mathrm{ex}}(\nu)\right] \bar{N}_{\mathrm{S} 1, l}\left(r, t_{\mathrm{d}}\right)\right\} I_{\mathrm{P}}\left(\nu, r, t^{\prime}\right) .
\end{aligned}
$$

The solution of eq. (12) gives the spectrally resolved probe pulse intensity transmission

$T_{\mathrm{P}}\left(\nu, r, t^{\prime}\right)=\frac{I_{\mathrm{P}}\left(\nu, r, t^{\prime}, l\right)}{I_{\mathrm{P}}\left(\nu, r, t^{\prime}, 0\right)}=T_{\mathrm{a}}(\nu) \exp \left\{\left[\sigma_{\mathrm{a}}(\nu)+\sigma_{\mathrm{e}}(\nu)-\sigma_{\mathrm{ex}}(\nu)\right] \bar{N}_{\mathrm{S} 1, l}\left(r, t_{\mathrm{d}}\right) l\right\}$,

where $T_{\mathrm{a}}(\nu)=\exp \left[-\sigma_{\mathrm{a}}(\nu) N_{0} l\right]$ is the small-signal transmission of the sample at frequency $\nu=c_{0} \tilde{\nu}=c_{0} / \lambda . T_{\mathrm{P}}(\nu$, $\left.r, t^{\prime}\right)$ is equal to the time-integrated probe pulse transmission $T_{\mathrm{T}, \mathrm{P}}(\nu, r)=\int_{-\infty}^{\infty} I_{\mathrm{P}}\left(\nu, r, t^{\prime}, l\right) \mathrm{d} t^{\prime} / \int_{-\infty}^{\infty} I_{\mathrm{P}}\left(\nu, r, t^{\prime}\right.$, $0) \mathrm{d} t^{\prime}$ since the probe pulse does not change noticably the $S_{1}$-state level population and the probe pulse duration, $\Delta t_{\mathrm{P}} \approx \Delta t_{\mathrm{L}}$, is short compared to the $\mathrm{S}_{1}$-state relaxation constant $\tau_{\mathrm{F}}$. The spectral energy transmission of the probe pulse spectrum is

$$
\begin{aligned}
& T_{\mathrm{E}, \mathrm{P}}(\nu)=\frac{\int_{0}^{\infty} r \int_{-\infty}^{\infty} I_{\mathrm{P}}\left(\nu, r, t^{\prime} . l\right) \mathrm{d} t^{\prime} \mathrm{d} r}{\int_{0}^{\infty} r \int_{-\infty}^{\infty} I_{\mathrm{P}}\left(\nu, r, t^{\prime}, 0\right) \mathrm{d} t^{\prime} \mathrm{d} r}=\frac{\int_{0}^{\infty} r \exp \left(-r^{2} / r_{\mathrm{P}}^{2}\right) T_{\mathrm{T}, \mathrm{P}}(\nu, r) \mathrm{d} r}{\int_{0}^{\infty} r \exp \left(-r^{2} / r_{\mathrm{P}}^{2}\right) \mathrm{d} r} \\
& \approx T_{\mathrm{a}}(\nu) \exp \left\{\left[\sigma_{\mathrm{a}}(\nu)+\sigma_{\mathrm{e}}(\nu)-\sigma_{\mathrm{ex}}(\nu)\right] \bar{N}_{\mathrm{S} 1, l}\left(t_{\mathrm{d}}\right) l\right\},
\end{aligned}
$$

with

$\bar{N}_{\mathrm{S} 1, l}\left(t_{\mathrm{d}}\right)=\frac{\int_{0}^{\infty} r \exp \left(-r^{2} / r_{\mathrm{P}}^{2}\right) \bar{N}_{\mathrm{S} 1, l}\left(r, t_{\mathrm{d}}\right) \mathrm{d} r}{\int_{0}^{\infty} r \exp \left(-r^{2} / r_{\mathrm{P}}^{2}\right) \mathrm{d} r}=\frac{\int_{0}^{\infty} r \exp \left(-r^{2} / r_{\mathrm{P}}^{2}\right) \bar{N}_{\mathrm{S} 1, l}\left(r, t_{\mathrm{d}}\right) \mathrm{d} r}{\pi r_{\mathrm{P}}^{2}}$.

$\bar{N}_{\mathrm{S} 1, l}\left(t_{\mathrm{d}}\right)$ is the effective $\mathrm{S}_{1}$-state level population density which is averaged over the molecular orientations, the sample length, and the radial probe pulse extension. In eq. (14) the approximation is reasonably accurate in cases of $0.2 \leqslant T_{\mathrm{E}, \mathrm{P}}(\nu) / T_{\mathrm{a}}(\nu) \leqslant 5$ (linear expansion of exponential function) or $r_{\mathrm{P}} / r_{\mathrm{L}} \leqslant 0.3\left(\bar{N}_{\mathrm{S} 1}\left(r, t_{\mathrm{d}}\right)\right.$ is rather constant within radial probe pulse extension, see below figs. 4 and 5 ). The approximation of eq. (14) allows the direct determination of the excited-state absorption cross section 
$\sigma_{\mathrm{ex}}(\nu) \approx-\frac{\ln \left[T_{\mathrm{E}, \mathrm{P}}(\nu) / T_{\mathrm{a}}(\nu)\right]}{\bar{N}_{\mathrm{S} 1, l}\left(t_{\mathrm{d}}\right) l}+\sigma_{\mathrm{a}}(\nu)+\sigma_{\mathrm{e}}(\nu)$

Below eq. (16) is used to determine $\sigma_{\mathrm{ex}}(\nu)$ from the measured probe pulse transmission $T_{\mathrm{E}, \mathrm{P}}(\nu)$, the calculated effective $S_{1}$-state level population $\bar{N}_{\mathrm{S} 1, l}\left(t_{\mathrm{d}}, T_{\mathrm{E}, \mathrm{L}}\right)$, and the known absorption cross-section spectrum $\sigma_{\mathrm{a}}(\nu)$ and stimulated emission cross-section spectrum $\sigma_{\mathrm{e}}(\nu)$ [32].

It should be noted that a sample is a potential laser medium in the spectral region where $T_{\mathrm{E}, \mathrm{P}}(\nu)>1$, i.e. $\sigma_{\mathrm{e}}(\nu)>\sigma_{\mathrm{ex}}(\nu)$, it is a potential saturable absorber in the region where $T_{\mathrm{a}}(\nu)<T_{\mathrm{E}, \mathrm{P}}(\nu)<1$, i.e. $\sigma_{\mathrm{ex}}(\nu)<\sigma_{\mathrm{a}}(\nu)$, and it is a potential reverse saturable absorber in the frequency range where $T_{\mathrm{E}, \mathrm{P}}(\nu)<T_{\mathrm{a}}(\nu)$, i.e. $\sigma_{\mathrm{ex}}(\nu)>\sigma_{\mathrm{a}}(\nu)$.

In the derivation of $\bar{N}_{\mathrm{S} 1, l}\left(t_{\mathrm{d}}\right)$ the amplified spontaneous emission has not been included. Its influence is estimated in the following. The reduction factor $f=\bar{N}_{\mathrm{S} 1, l, A \mathrm{ASE}}\left(t_{\mathrm{d}}\right) / N_{\mathrm{S} 1, l, 0}\left(t_{\mathrm{d}}\right)$ is equal to $\tau_{\mathrm{ASE}} / \tau_{\mathrm{F}}$, where $\bar{N}_{\mathrm{S} 1, l, \mathrm{ASE}}\left(t_{\mathrm{d}}\right)$ is the $\mathrm{S}_{1}$-state level population at time $t_{\mathrm{d}}$ including amplified spontaneous emission, $\bar{N}_{\mathrm{S} 1, l, 0}\left(t_{\mathrm{d}}\right)$ is the $\mathrm{S}_{1}$-state level population without amplified spontaneous emission, and $\tau_{\text {ASE }}$ is the $S_{1}$-state lifetime in the case of amplified spontaneous emission. The reduction of the $S_{1}$-state lifetime by amplified spontaneous emission was derived in refs. [33,34]. It reads

$\frac{\tau_{\mathrm{ASE}}}{\tau_{\mathrm{F}}} \approx \frac{1}{1+q_{\mathrm{F}}(\Delta \Omega / 4 \pi) T_{\mathrm{E}, \mathrm{P}}\left(\nu_{\mathrm{ASE}}\right)}$,

where $q_{\mathrm{F}}$ is the fluorescence quantum yield, $\Delta \Omega=\pi r_{\mathrm{L}}^{2} / l^{2}$ is the solid angle of effective amplified spontaneous emission, and $\nu_{\text {ASE }}$ the amplified spontaneous emission frequency (positions of maximum gain $T_{E, P}$ ). For our experimental situation $\left(r_{\mathrm{L}} \approx 1 \mathrm{~mm}, l=1 \mathrm{~cm}, q_{\mathrm{F}}=0.44, T_{\mathrm{E}, \mathrm{P}}\left(\nu_{\mathrm{ASE}}\right) \approx 7\right)$ we estimate $\tau_{\mathrm{ASE}} / \tau_{\mathrm{F}} \approx 0.99$.

\section{Determination of $S_{1}$-state population}

The spectroscopic parameters of eosin $\mathrm{Y}$ in methanol which are applied in the calculations are collected in table 1 . The pump pulse energy transmission $T_{\mathrm{E}, \mathrm{L}}$ and the normalized length and orientation averaged $\mathrm{S}_{1}$-state level population $\bar{N}_{\mathrm{S} 1, l}\left(r=0, t_{\mathrm{d}}=30\right.$ ps) $/ N_{0}$ versus input pump pulse peak intensity $I_{0 L}$ are plotted in fig. 3. At high pump pulse intensities $\left(I_{0 \mathrm{~L}} \rightarrow \infty\right)$ a finite ground-state population $N_{1, \infty}=$ $N_{2^{*}, \infty} \approx\left(N_{0}-N_{1, \infty}\right) \exp \left(-h \Delta \nu_{2,2^{*}} / k_{\mathrm{B}} \vartheta\right)$ remains because of the thermal population of the excited Franck-Condon state within the $S_{1}$ band. The re-

Table 1

Parameters of eosin $\mathrm{Y}$ in mèthanol at room temperature

\begin{tabular}{lll}
\hline Parameter & Value & Reference \\
\hline$\tau_{\mathrm{F}}$ & $2.0 \mathrm{~ns}$ & {$[32]$} \\
$\tau_{\text {or }}$ & $330 \mathrm{ps}$ & {$[51]$} \\
$\tau_{\mathrm{FC}}$ & $0.7 \mathrm{ps}$ & assumed, [52] \\
$\tau_{\mathrm{ex}}$ & $60 \mathrm{fs}$ & assumed, [53] \\
$\Delta \tilde{v}_{2,2 \bullet}$ & $240 \mathrm{~cm}^{-1}$ & {$[32]$} \\
$\sigma_{\mathrm{ex}, \mathrm{L}}$ & $(3 \pm 0.5) \times 10^{-17} \mathrm{~cm}^{2}$ & {$[32]$} \\
$q_{\mathrm{F}}$ & 0.44 & {$[32]$} \\
\hline
\end{tabular}

maining ground-state population $N_{1, \infty} \approx N_{0} \times$ $\exp \left(-h \Delta \nu_{2,2 *} / k_{\mathrm{B}} \vartheta\right) /\left[1+\exp \left(-h \Delta \nu_{2,2^{*}} / k_{\mathrm{B}} \vartheta\right)\right]$ reduces the maximum length and orientation averaged

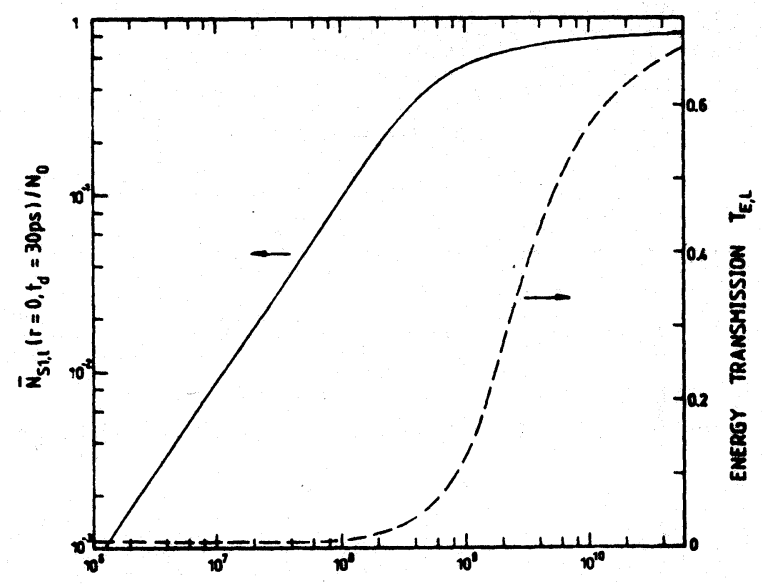

INPUT PEAK INTENSITY $t_{\alpha} \quad\left(W \mathrm{~cm}^{-2}\right)$

Fig. 3. Pump pulse energy transmission $T_{E . L}$ (dashed curve, $\lambda_{L}=527 \mathrm{~nm}$ ) and normalized length and orientation averaged $S_{1}$-state level population $\vec{N}_{\mathrm{St}, l}\left(r=0, t_{\mathrm{d}}=30 \mathrm{ps}\right) / N_{0}$ (solid curve) versus pump pulse input peak intensity $I_{0 L}$. Curves are calculated by solving the equation system 1 to 5 with the parameters of table 1. 


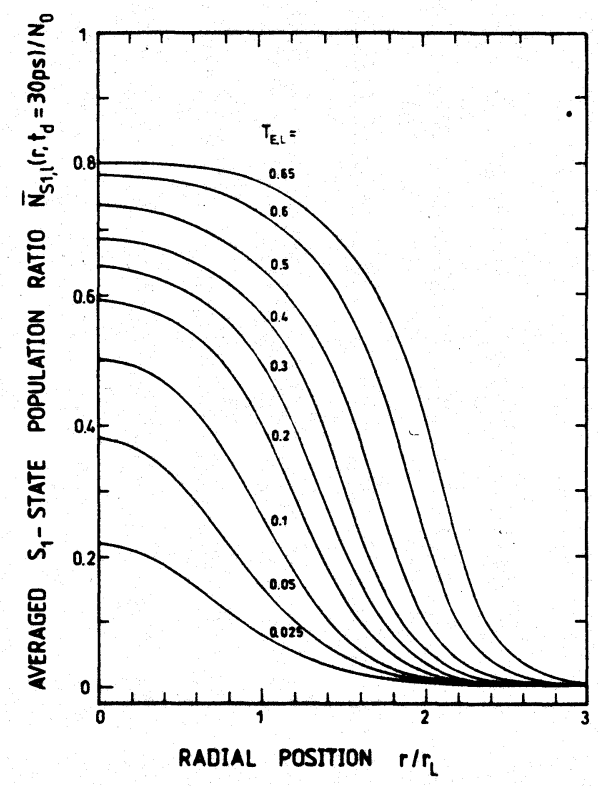

Fig. 4. Normalized orientation and length averaged $S_{1}$-state level population $\bar{N}_{\mathrm{sl}, l}\left(r=0, t_{\mathrm{d}}=30 \mathrm{ps}\right) / N_{0}$ versus normalized radial pump pulse position $r / r_{\mathrm{L}}$. The curves belong to the indicated pump pulse energy transmissions $T_{E, L}$. The curves are obtained from fig. 3 by using a Gaussian beam profile.

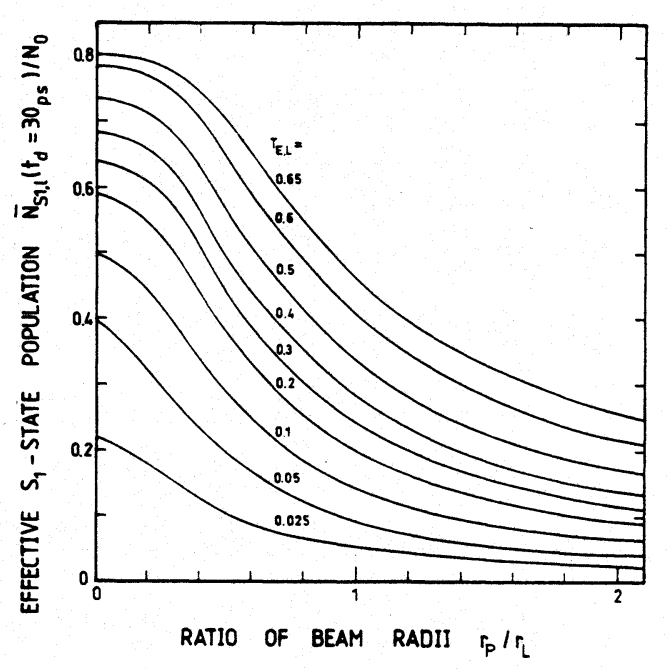

Fig. 5. Normalized effective orientationally, longitudinally and transversally averaged $\mathrm{S}_{1}$-state level population $\bar{N}_{\mathrm{S}, l}\left(t_{\mathrm{d}}=30 \mathrm{ps}\right)$ versus the ratio of pump pulse to probe pulse beam radius $r_{\mathrm{p}} / r_{\mathrm{L}}$ for Gaussian beam profiles. The various curves belong to the indicated pump pulse energy transmissions $T_{\mathrm{E}, \mathrm{L}}$.
$\mathrm{S}_{1}$-state level population to $\bar{N}_{\mathrm{S} 1, l, \max } \approx N_{0} /[1+$ $\left.\exp \left(-h \Delta \nu_{2.2^{*}} / k_{\mathrm{B}} \vartheta\right)\right]$.

In fig. 4 the normalized length and orientation averaged $\mathrm{S}_{1}$-state level population $\bar{N}_{\mathrm{S} 1, l}\left(r, t_{\mathrm{d}}=30 \mathrm{ps}\right) /$ $N_{0}$ is plotted versus the normalized pump beam radius $r / r_{\mathrm{L}}$ for various fixed pump pulse energy transmissions $T_{E, L}$. In fig. 5 the normalized effective $S_{1}$ state level population $\bar{N}_{\mathrm{S} 1, l}\left(t_{\mathrm{d}}=30 \mathrm{ps}\right) / N_{0}$ versus the ratio of probe pulse to pump pulse beam radius $r_{\mathrm{P}} / r_{\mathrm{L}}$ is displayed for various fixed pump pulse energy transmission $T_{\mathrm{E}, \mathrm{L}}$. This plot is applied in the $\sigma_{\mathrm{ex}}$ determination.

\section{Determination of $S_{1}$-excited-state absorption cross-section spectrum}

The ground-state absorption cross-section spectrum $\sigma_{\mathrm{a}}(\lambda)$ and the stimulated emission cross-section spectrum $\sigma_{\mathrm{e}}(\lambda)$ of eosin $\mathrm{Y}$ in methanol have been determined previously [32]. $\sigma_{\mathrm{a}}(\lambda)$ was obtained from transmission measurements in a spectrophotometer, and $\sigma_{\mathrm{e}}(\lambda)$ was determined from fluorescence quantum distribution measurements. The $\sigma_{\mathrm{a}}(\lambda)$ and $\sigma_{\mathrm{e}}(\lambda)$ curves are redrawn in fig. 7 . In the experimental studies the small signal transmission at the pump laser wavelength $\lambda_{\mathrm{L}}=527 \mathrm{~nm}$ was set to $T_{0}=T_{\mathrm{a}}\left(\lambda_{\mathrm{L}}\right)$ $=\exp \left(-N_{0} \sigma_{\mathrm{L}} l\right)=0.01 \quad\left(l=1 \quad \mathrm{~cm}, \quad \sigma_{\mathrm{L}}=3.2 \times 10^{-16}\right.$ $\left.\mathrm{cm}^{2}, N_{0}=1.44 \times 10^{16} \mathrm{~cm}^{-3}\right)$. The wavelength dependent ground-state small signal transmission $T_{\mathrm{a}}(\lambda)=\exp \left[-N_{0} \sigma_{\mathrm{a}}(\lambda) l\right]$ is shown by the shortdashed curve $T_{\mathrm{a}}$ in fig. 6 . The maximum amplification $T_{\mathrm{e}}\left(\lambda_{\mathrm{i}}\right)$ in the case of total population transfer to the $S_{1}$ state and in the absence of excited-state absorption would be $T_{\mathrm{e}}(\lambda)=\exp \left[N_{0} \sigma_{\mathrm{e}}(\lambda) l\right] . T_{\mathrm{e}}(\lambda)$ is displayed in fig. 6 by the short-dashed curve $T_{e}$.

The actual spectral energy transmission $T_{\mathrm{E}, \mathrm{P}}(\nu)$ of the probe pulse continuum is determined by the spectral probe pulse signals $S_{\text {out }}(\nu)$ behind the sample (registered with spectrometer SP2 and vidicon system VI) and $S_{\text {in }}(\nu)$ in front of the sample (registered with spectrometer SP1 and diode array system DA). It is

$$
\begin{aligned}
& T_{\mathrm{E}, \mathrm{P}}(\nu) \\
& =\frac{\left[S_{\text {out.d }}(\nu)-S_{\text {out }, \mathrm{b}}(\nu)\right] /\left[S_{\mathrm{in}, \mathrm{d}}(\nu)-S_{\mathrm{in}, \mathrm{b}}(\nu)\right]}{\left[S_{\text {out }, \mathrm{s}}(\nu)-S_{\text {out }, \mathrm{b}}(\nu)\right] /\left[S_{\mathrm{in}, \mathrm{s}}(\nu)-S_{\mathrm{in}, \mathrm{b}}(\nu)\right]},
\end{aligned}
$$




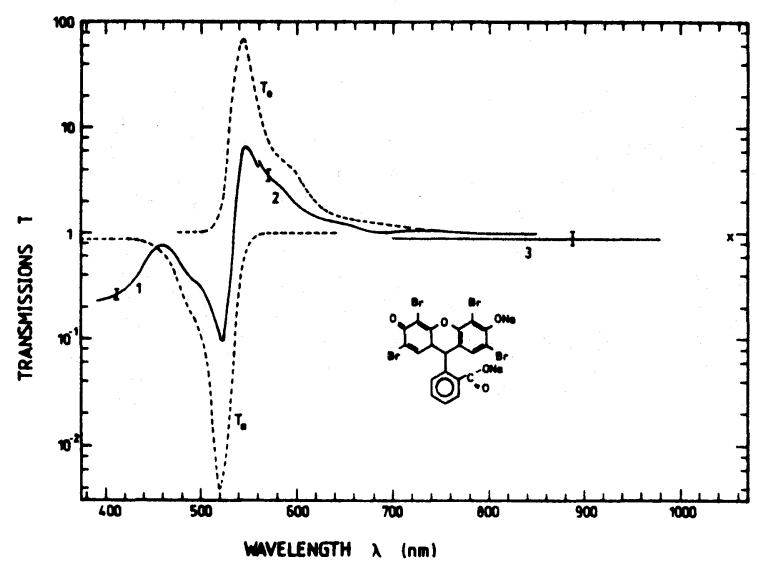

Fig. 6. Spectral transmission curves through eosin $Y$ in methanol. Sample length $l=1 \mathrm{~cm}$. Small signal transmission at pump laser wavelength of $\lambda_{\mathrm{L}}=527 \mathrm{~nm}$ is $T_{0}=\exp \left(-N_{0} \sigma_{\mathrm{L}} l\right)=0.01$. Short dashed curve $T_{a}$ gives small signal transmission $T_{\mathrm{a}}(\lambda)=\exp \left[-N_{0} \sigma_{\mathrm{a}}(\lambda) l\right]$. Short-dashed curve $T_{\mathrm{e}}$ represents $T_{e}(\lambda)=\exp \left[N_{0} \sigma_{e}(\lambda) l\right]$. The solid curves show examples of delayed picosecond probe continuum transmissions $\left(t_{\mathrm{d}} \approx 30 \mathrm{ps}\right)$ in different spectral regions. They belong to pump pulse transmissions of (1) $T_{E, L}=0.48$, (2) $T_{E, L}=0.32$, (3) $T_{E, L}=0.3$. The structural formula of eosin $\mathrm{Y}$ is inserted.

where the indices $d, s$, and $b$ indicate the signals obtained with the eosin $Y$ dye solution, the solvent methanol, and the background detector signal, respectively.

The solid curves 1, 2, 3 in fig. 6 show three spectra obtained in different spectral regions by single laser shots. The pump pulse energy transmissions for these three examples where $T_{\mathrm{E}, \mathrm{L}}(1)=0.48, T_{\mathrm{E}, \mathrm{L}}(2)=0.32$, and $T_{\mathrm{E}, \mathrm{L}}(3)=0.30$. The ratio of the beam radii at the sample position was $r_{\mathrm{P}} / r_{\mathrm{L}} \approx 0.32$. Applying fig. 5 the effective $S_{1}$-state level populations at the time $t_{\mathrm{d}}$ of the probe pulse passage are $\bar{N}_{S 1, l}(1) \approx 0.66 \times N_{0}$, $\bar{N}_{\mathrm{S} 1, l}(2) \approx 0.57 \times N_{0}$ and $\bar{N}_{\mathrm{S} 1, \ell}(3) \approx 0.56 \times N_{0}$. The corresponding excited-state absorption cross-section spectra are obtained from eq. (16).

The solid curve $\sigma_{\mathrm{ex}}$ shown in fig. 7 was obtained by averaging over three $\sigma_{\mathrm{ex}}(\nu)$ spectra obtained by the single-shot $T_{\mathrm{E}, \mathrm{P}}(\nu)$ and $T_{\mathrm{E}, \mathrm{L}}$ data analysis. In the wavelength region $400<\lambda \leqslant 520 \mathrm{~nm}$ there is no stimulated $S_{1}$-state emission contribution to the probe continuum transmission. In the wavelength region $520<\lambda<550 \mathrm{~nm}$ (dotted $\sigma_{\mathrm{ex}}$ curve) an accurate $\sigma_{\mathrm{ex}}$ determination was difficult because of the change from strong ground-state absorption to strong stimulated emission. The actual transmission $T_{\mathrm{E}, \mathrm{P}}$ be-

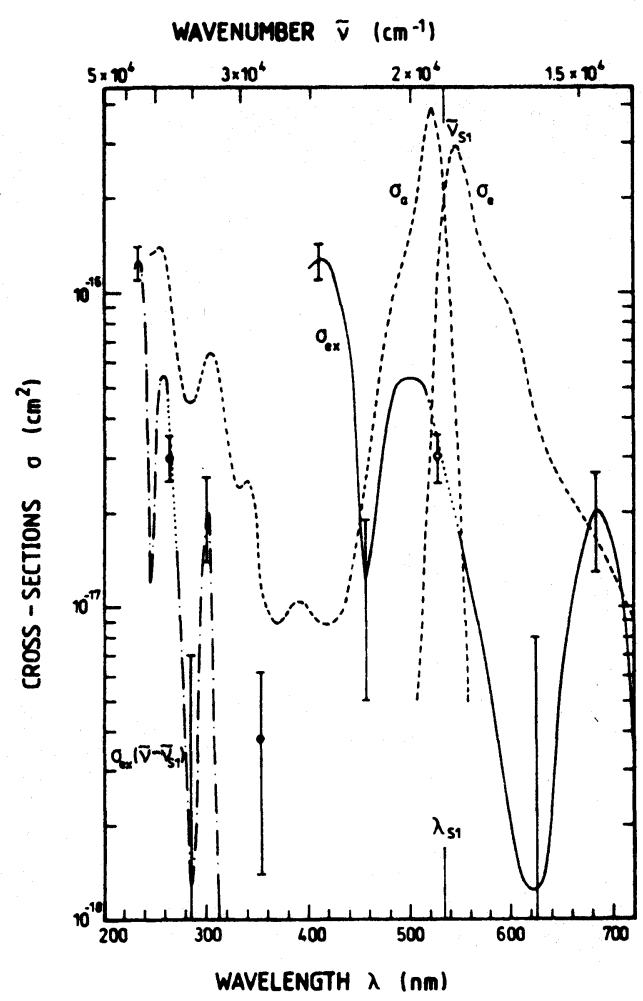

Fig. 7. Cross sections of eosin $\mathrm{Y}$ in methanol at room temperature. Dashed curves, $\sigma_{\mathrm{a}}(\lambda)$ and $\sigma_{\mathrm{e}}(\lambda)$. Solid curve and circle. $\sigma_{\text {ex }}(\lambda)$. Dash-dotted curve and dots, $\sigma_{\text {ex }}\left(\tilde{\nu}-\tilde{\nu}_{\mathrm{st}}\right)$ where $\tilde{\nu}$ is the probe wavenumber and $\tilde{\nu}_{\mathrm{S} 1}$ is the electronic $S_{0}-S_{1}$ transition wavenumber.

comes very sensitive to the actual $S_{1}$-state level population $\bar{N}_{\mathrm{s} 1,1}$. Additionally the absolute stimulated emission cross section $\sigma_{\mathrm{e}}(\nu)$ is not known exact enough to give exact $\sigma_{\text {ex }}(\nu)$ data. The light scattering at the pump laser wavelength $\lambda_{L}=527 \mathrm{~nm}$ aggrevates an accurate $\sigma_{\mathrm{ex}}$ determination around $\lambda_{\mathrm{L}}$. At $\lambda_{\mathrm{L}}$ the $S_{1}$-state excited-state absorption cross section has been determined previously by intensity dependent pump pulse transmission measurements $T_{\mathrm{E}, \mathrm{L}}\left(I_{\mathrm{OL}}\right)$ [32]. The obtained $\sigma_{\text {ex }}\left(\lambda_{L}\right)$ value is shown by a circle in fig. 7 . In the long-wavelength region $\lambda \gtrsim 550 \mathrm{~nm}$ the actual probe continuum transmission measurements determines $\sigma_{\mathrm{e}}(\lambda)-\sigma_{\mathrm{ex}}(\lambda)$, and the accuracy of $\sigma_{\mathrm{ex}}(\lambda)$ depends on the accuracy of $\sigma_{\mathrm{e}}(\lambda)$. The error bars shown on the $\sigma_{\mathrm{ex}}(\lambda)$ curve indicate the standard deviations of the three measurements. The minimum of $\sigma_{\mathrm{ex}}$ around $620 \mathrm{~nm}$ would be less pronounced if $\sigma_{\mathrm{e}}(\lambda)$ would be slightly larger than shown 
in fig. 7. For $\lambda \gtrsim 700 \mathrm{~nm}$ the stimulated emission cross section becomes small and $T_{\mathrm{E}, \mathrm{P}}$ becomes determined by $\sigma_{\mathrm{ex}}$. The measured probe continuum transmissions give $\sigma_{\mathrm{ex}} \leqslant 10^{-17} \mathrm{~cm}^{2}$ in the wavelength region $720<\lambda<980 \mathrm{~nm}$ which is not shown in fig. 7 (the spectra of ten laser shots were analysed). At $\lambda=1054$ $\mathrm{nm}$ the excited-state absorption cross section has been determined by measuring the transmission of the strongly attenuated time delayed fundamental laser pulses through the eosin $\mathrm{Y}$ sample after second harmonic light excitation instead of the transmission measurement of the light continuum. The obtained averaged energy transmission was $T_{\mathrm{E}}=0.973 \pm 0.017$ for $T_{\mathrm{E}, \mathrm{L}} \approx 0.2\left(r_{\mathrm{P}} / r_{\mathrm{L}} \approx 0.3, \bar{N}_{\mathrm{S} 1, l}\left(t_{\mathrm{d}}\right) \approx 0.50\right.$, average over 20 shots, result shown by the cross in fig. 6 ). The corresponding excited-state absorption cross section is $\sigma_{\mathrm{ex}}(1054 \mathrm{~nm})=(3.8 \pm 2.4) \times 10^{-18} \mathrm{~cm}^{2}$.

\section{Discussion}

The singlet excited-state absorption originates from the thermally relaxed population distribution in the $S_{1}$ state at a frequency $\nu_{\mathrm{S} 1}=c_{0} \nu_{\mathrm{S} 1}=c_{0} / \lambda_{\mathrm{S} 1}$. The dashdotted curve in fig. 7 presents the frequency shifted excited-state absorption cross-section curve $\sigma_{\text {ex }}(\tilde{\nu}-$ $\left.\tilde{\nu}_{\mathrm{S} 1}\right)$ versus $\tilde{\nu}$. The absorption maxima of the frequency shifted dash-dotted $\sigma_{\mathrm{ex}}\left(\tilde{\nu}-\tilde{\nu}_{\mathrm{S} 1}\right)$ curve and the absorption maxima of the short dashed ground-state absorption cross-section curve $\sigma_{\mathbf{a}}(\tilde{\nu})$ coincide reasonably well and correspond to the same higher excited singlet states $S_{n}(n \geqslant 2)$. In the displayed frequency range the $S_{1}-S_{n}$ excited-state absorption cross sections are smaller than the $S_{0}-S_{n}$ ground-state absorption cross sections.

A comparison of $\sigma_{\mathrm{ex}}(\lambda)$ with $\sigma_{\mathrm{a}}(\lambda)$ and $\sigma_{\mathrm{e}}(\lambda)$ shows that eosin $Y$ in methanol may be used as a reverse saturable absorber in the wavelength region of $400<\lambda<450 \mathrm{~nm}\left(\sigma_{\mathrm{ex}}>\sigma_{\mathrm{a}}\right.$, the short-wavelength range probably extends to $300 \mathrm{~nm}$ ), as a saturable absorber in the region $450<\lambda<550 \mathrm{~nm}\left(\sigma_{\mathrm{ex}}<\sigma_{\mathrm{a}}\right)$, and as a laser gain medium in the wavelength range $530 \leqslant \lambda \leqslant 670 \mathrm{~nm}\left(\sigma_{\mathrm{ex}}<\sigma_{\mathrm{e}}\right)$. The use of eosin $\mathrm{Y}$ in methanol as a laser medium may be restricted to short pump pulse excitation ( $p$ s to ns) and subpicosecond to subnanosecond laser pulse generation [35] like travelling-wave amplified spontaneous emission lasers [33.35-40], distributed feedback lasers [41,42], quenched cavity lasers [43,44], spectro-temporal selection lasers [45,46], or short cavity lasers [4650 ], because of the large quantum yield of triplet state formation [32].

\section{Conclusions}

The absolute singlet excited-state absorption crosssection spectrum of eosin $\mathrm{Y}$ in methanol has been determined in the wavelength regions $400 \leqq \lambda \lesssim 520 \mathrm{~nm}$, $550 \lesssim \lambda \lesssim 980 \mathrm{~nm}$, and at $\lambda=527 \mathrm{~nm}$ and $\lambda=1054 \mathrm{~nm}$. A picosecond pump (second harmonic pulses of a mode-locked Nd:glass laser) and probe (picosecond light continuum generated in $\mathrm{D}_{2} \mathrm{O}$ ) technique was applied. The $S_{1}-S_{n}$ excited-state absorption crosssection spectrum is found to be roughly a factor of three weaker than the $S_{0}-S_{n}$ ground-state absorption cross-section spectrum. A comparison of the $S_{1}$-excited-state absorption cross-section spectrum with the $S_{0}$-ground-state absorption cross-section spectrum and the $S_{1}$ stimulated emission cross-section spectrum allowed the localization of the spectral regions where the dye solution may be applied as a reverse saturable absorber (laser induced absorption enhancement), a saturable absorber (laser induced absorption reduction), or a laser gain medium (gain dominates over absorption ).

\section{References}

[1] G.R. Fleming, Chemical applications of ultrafast spectroscopy (Oxford Univ. Press, Oxford, 1986).

[2] W. Kaiser, ed., Topics in applied physics, Vol. 60. Ultrashort laser pulses and applications (Springer, Berlin, 1988).

[3] V. Brückner, K.H. Feller and U.W. Grummet, Applications of time-resolved optical spectroscopy (Elsevier, Amsterdam, 1990).

[4] S. Mukamel, Ann. Rev. Phys. Chem. 41 (1990) 647.

[5] L.R. Khundkar and A.H. Zewail, Ann. Rev. Phys. Chem. $41(1990) 15$

[6] I.A. Walmsley and J.D. Kafka, in: Contemporary nonlinear optics, eds. G.P. Agrawal and R.W. Boyd (Academic Press, New York, 1992) p. 119.

[7] M.A. Vasil'eva, A.V. Masalov, J. Vishchakas, V. Gulbinas, V. Kabelka and V. Syrus, Appl. Phys. B 37 (1985) 41.

[8] J. Glownia, J. Misewich, R. Walkup, M. Kaschke and P. Sorokin, in: Topics in applied physics, Vol. 70. Dye lasers: 25 years, ed. M. Stuke (Springer, Berlin, 1992) p. 1. 
[9] T.H. Wei, D.J. Hagan, M.J. Seuce, E.W. VanStryland, J.W. Perry and D.R. Coulter, Appl. Phys. B 54 (1992) 46.

[10] R. Dorsinville, P.P. Ho, J.T. Manassah and R.R. Alfano, in: The supercontinuum laser source, ed. R.R. Alfano (Springer, Berlin, 1989) p. 377.

[11] Y.B. Band and R. Bavli, Phys. Rev. A 36 (1987) 3203.

[12] M.J. Wirth, Anal. Chem. 62 (1990) 270A.

[13]E. Göbel, in: Advances in Solid State Physics, Vol. 30. Festkörperprobleme, ed. U. Rössler (Vieweg, Braunschweig, 1990) p. 269.

[14] S. Speiser, Chem. Phys. 6 (1974) 479.

[15] S. Speiser and A. Bromberg, Chem. Phys. 9 (1975) 191.

[16] K. Dasgupta and L.G. Nair, IEEE J. Quantum Electron. 26 (1990) 189.

[17] A. Penzkofer, Appl. Phys. B 46 (1988) 43.

[18] S. Speiser, R. van der Werf and J. Kommandeur, Chem. Phys. 1 (1973) 297.

[19] S. Speiser, Opt. Commun. 45 (1983) 84.

[20] S. Speiser and N. Shakour, Appl. Phys. B 38 (1985) 159.

[21] Y.B. Band, Phys. Rev. A 34 (1986) 326.

[22] W. Blau, H. Byrne, W.M. Dennis and J.M. Kelly, Opt. Commun. 56 (1985) 25.

[23] A.V. Deshpande and N.B. Iyer, J. Luminescence 46 (1990) 339.

[24] D. Wittekind, in: Standardization and quantization of diagnostic staining in cytology, eds. M.E. Born and L.P. Kok (Coulomb Press, Leiden, 1986) p. 30.

[25] S. Speiser and F.L. Chisena, J. Chem. Phys. 89 (1988) 7259.

[26] K.W. Beeson, J.T. Yardley and S. Speiser, Mol. Eng. 1 (1991) 1.

[27] A. Penzkofer and F. Graf, Opt. Quant. Electron. 17 (1985) 219.

[28] A. Penzkofer and W. Kaiser, Opt. Quant. Electron. 9 ( 1977) 315.

[29] D. von der Linde, O. Bernecker and A. Laubereau, Opt. Commun. 2 (1970) 215.

[30] V.G. Dimitriev, G.G. Gurzadyan and D.N. Nikogosyan, Springer series in optical sciences, Vol. 64. Handbook of nonlinear optical crystals (Springer, Berlin, 1991) p. 65.

[31] E.D. Cehelnik, K.D. Mielenz and R.A. Velagoldi, J. Res. Natl. Bur. Std. A 79 (1975) 1.
[32] A. Penzkofer, A. Beidoun and M. Daiber, J. Luminescence 51 (1992) 297.

[33] A. Penzkofer and W. Falkenstein, Opt. Quant. Electron. 10 (1978) 399.

[34] A. Penzkofer and W. Blau, Opt. Quant. Electron. 15 (1983) 325.

[35] F.P. Schäfer, in: Topics in applied physics, Vol. 70. Dye lasers: 25 years, ed. M. Stuke (Springer, Berlin, 1992) p. 19.

[36] Zs. Bor, S. Szatmári and A. Müller, Appl. Phys. B 32 (1983) 101.

[37] F. Wondrazek, A. Seilmeier and W. Kaiser, Appl. Phys. B 32 (1983) 39.

[38] P. Sperber, W. Spangler, B. Meier and A. Penzkofer, Opt. Quant. Electron. 20 (1988) 395.

[39] P. Qiu and A. Penzkofer, Appl. Phys. B 48 (1989) 115.

[40] J. Hebling and J. Kuhl, Opt. Commun. 73 (1989) 375.

[41] Zs. Bor, IEEE J. Quant. Electron. QE-16 (1980) 517.

[42] S. Szatmári and F.P. Schäfer, Appl. Phys. B 46 (1988) 305.

[43] A. Eranian, P. Dezausier and O. de Witte, Opt. Commun. 7 (1973) 150.

[44] F.P. Schäfer, L. Wenchong and S. Szatmári, Appl. Phys. B 32 (1983) 123.

[45] M.M. Martin, E. Breheret and Y.H. Meyer, Opt. Commun. 56 (1985) 61

[46] N. Dai Hung, P. Plaza, M. Martini and Y.H. Meyer, Appl. Opt. 31 (1992) 7046.

[47] B. Fan and T.K. Gustafson, Appl. Phys. Letters 28 (1976) 202.

[48] A.J. Cox, C.D. Merrit and G.W. Scott, Appl. Phys. Letters 40 (1982) 664.

[49] G.W. Scott, S.G. Shen and A.J. Cox, Rev. Sci. Instr. 55 (1984) 358.

[50] Y. Kawakatsu, K. Miyazaki, T. Hasama and T. Sato, Appl. Opt. 25 (1986) 634.

[51] G.R. Fleming, J.M. Morris and G.W. Robinson, Chem. Phys. 17 (1976) 91.

[52]A. Penzkofer, W. Falkenstein and W. Kaiser, Chem. Phys. Letters 44 (1976) 82.

[53] F. Graf and A. Penzkofer, Opt. Quant. Electron. 17 (1985) 53. 